\title{
Long-term Starspot Behavior of BY Dra and EV Lac
}

\author{
B.R. Pettersen ${ }^{1}$, K. Oláh ${ }^{2}$, and W.H. Sandmann ${ }^{3}$ \\ ${ }^{1}$ Institute of Theoretical Astrophysics, University of Oslo, \\ P.O.Box 1029 Blindern, N-0315 Oslo 3, Norway \\ ${ }^{2}$ Konkoly Observatory, Hungarian Academy of Sciences \\ P.O.Box 67, H-1525 Budapest XII, Hungary \\ ${ }^{3}$ Department of Physics, Harvey Mudd College, \\ Claremont, CA 91711, USA
}

\begin{abstract}
New starspot photometry of BY Dra and EV Lac, extending over a decade is analyzed in combination with data from the literature to study the long-term behaviour of spottedness on these stars. Both stars show brightness suppression as well as brightness modulation so spots exist both near the poles and at lower latitudes. Polar spots have been continuously present on BY Dra for the last 14 years. $(\mathrm{O}-\mathrm{C})$ diagrams for lightcurve minima reveal systematic, sinusoidal variations in BY Dra with a period of 13-14 years. In EV Lac the spots have been located on the same hemisphere for the last ten years.
\end{abstract}

\section{Introduction and observations}

New photometric observations of the binary BY Dra and the single star EV Lac have been obtained during the last decade. We analyse them in combination with previously published data to determine long-term properties of spottedness.

The observations were made with various combinations of telescopes and photometers at different observatories: the Piszkéstető observing station of Konkoly Observatory in Hungary, McDonald Observatory, Texas, and Table Mountain Observatory, California, USA.

\section{Results and discussion}

BY Dra. The brightness level and range of variability for BY Dra is shown as a function of time in the upper part of Fig. 1. When rotational modulation due to starspots was discovered by Chugainov (1973) the brightness suppression and amplitude were at record values (in 1965). The large brightness suppression is presumably due to a polar spot on the primary star visible at all rotational phases, since $i \approx 30^{\circ}$. The brightness modulation signifies a large spot at lower latitudes that rotates in and out of view. It could have been located on either of the two stars. When these spots decayed in area during 1967-69 the brightness level of BY 


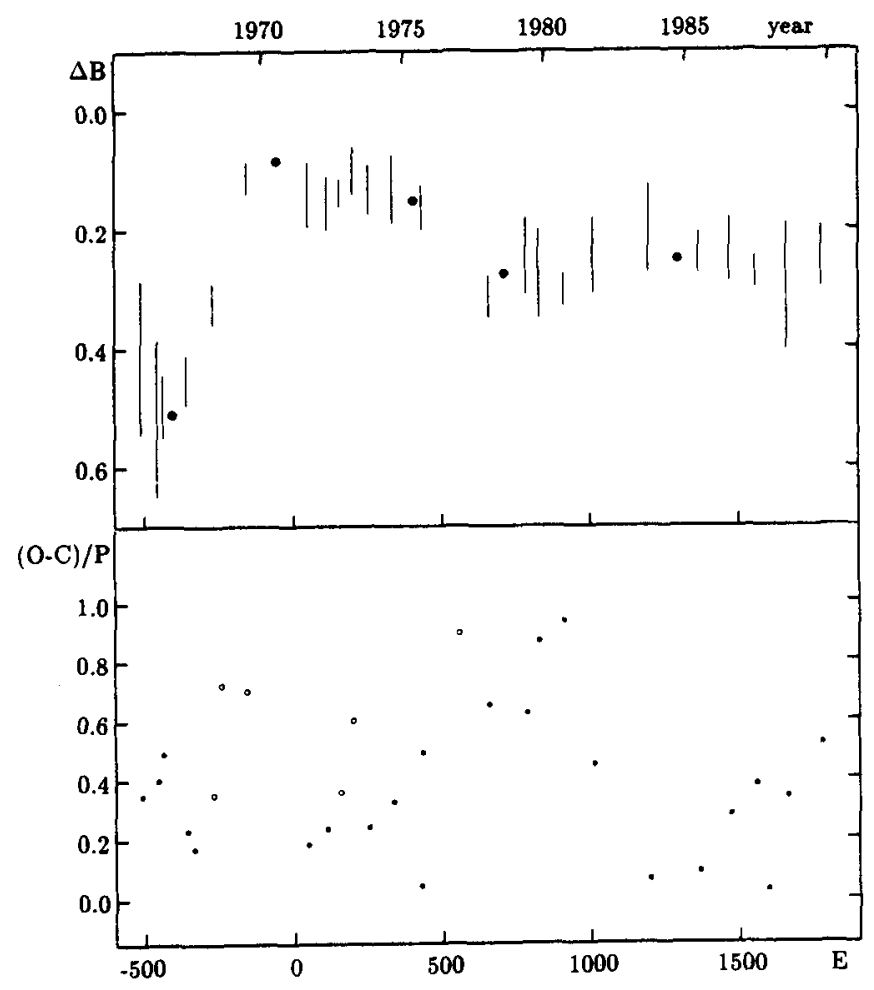

Fig. 1. Seasonal brightness changes of BY Dra from 1965 to 1989 (upper panel). The length of each bar represents the range of brightness variations. Episodes of flat lightcurves are shown as filled circles. The lower panel shows the (O-C) diagram for the same time interval, with uncertain minima identified by open circles. The sinusoidal variation of the accurate determinations (filled circles) has a period of 13-14 years.

Dra gradually increased to the immaculate level. This level seems to have been maintained for several years. The lack of $\mathrm{H} \alpha$ emission from the primary in 1974 suggests that this star had entered a state of no or very little atmospheric activity while the secondary remained active with $\mathrm{H} \alpha$ in emission. A year later the primary developed a weak emission which over the next two years grew to match that of the secondary in 1977 (Vogt, 1980). During this development a new episode of brightness suppression began, presumably due to new polar spots on the primary. 14 years later, in 1989 , this state still persisted.

We have used the average period ( $P=3.8285$ days) for the entire $1965-1989$ dataset to produce a linear ephemeris for the stellar rotation.

For each lightcurve we compare the observed time of minimum with the computed time and plot $\frac{(O-C)}{P}$ versus time (Figure 1, lower panel). The general trend 
is that $\frac{(O-C)}{P}$ varies in a sinusoidal fashion with a period of 13-14 years. The peak-to-peak amplitude is at least 5 times the typical scatter of datapoints.

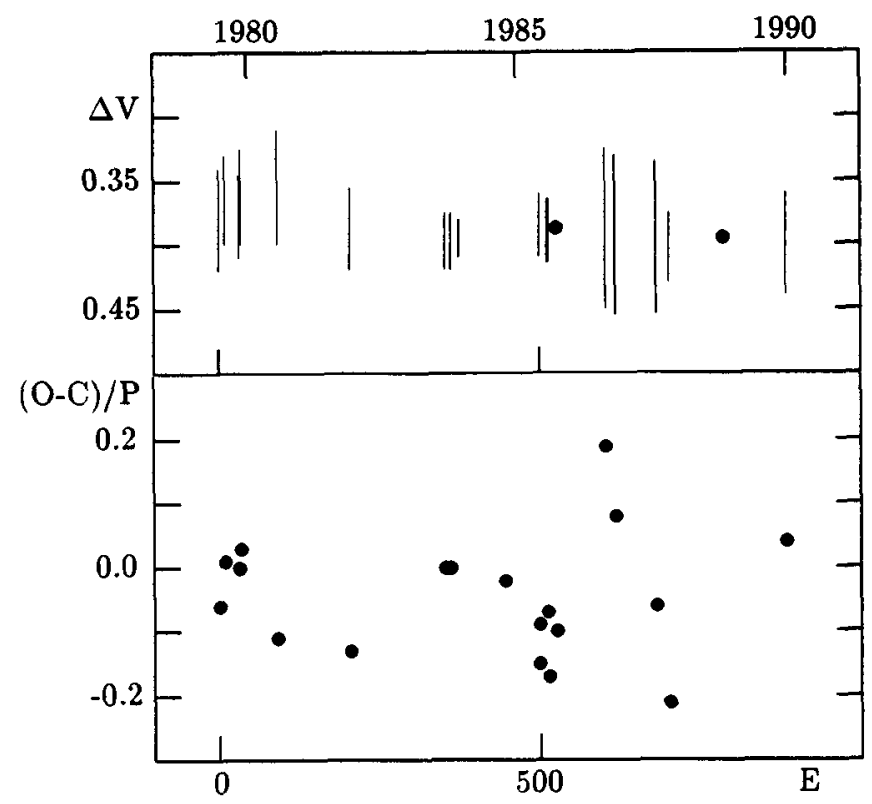

Fig. 2. The brightness changes of EV Lac from 1979 to 1990 (upper panel). Episodes of flat lightcurves are identified by filled circles. The $(\mathrm{O}-\mathrm{C})$ diagram (lower panel) demonstrates that spots were located on the same hemisphere of the star during the entire decade.

EV Lac. The brightness of EV Lac usually shows variations less than $0.1 \mathrm{mag}$. Small suppressions of the kind produced by polar spots are present during most of the past decade. Times of minima can be read off directly from the lightcurves and are used to form the $(\mathrm{O}-\mathrm{C})$ difference relative to a linear ephemeris with a period of 4.376 days (Fig. 2). The plot of $\frac{(O-C)}{P}$ versus time shows lightcurve minima to occur within 0.2 of the same phase during the entire decade. That means that spots were located preferentially on one hemisphere of EV Lac during a ten year interval.

\section{References}

Chugainov, P.F.: 1973, Izv. Krymsk. Astrofiz. Obs. 48, 3

Vogt, S.S.: 1980, Astrophys. J. 240, 567 